\title{
DC Conductivity Measurements in the Van Der Pauw Geometry
}

\author{
Gert Rietveld, Ch. V. Koijmans, Lesley C. A. Henderson, M. J. Hall, S. Harmon, Peter Warnecke, and \\ Bernd Schumacher
}

\begin{abstract}
A new methodology for conductivity measurements, where square metallic samples are measured with the Van der Pauw technique, has been successfully implemented. The uncertainty obtained is $0.04 \%$ and a comparison between national metrology institutes gives an agreement of the measurement values within $0.035 \%$. Major advantages of the new method are that smaller reference samples are required and only a single dimensional measurement is needed.
\end{abstract}

Index Terms-Calibration, conductivity, direct current, eddy current, International Annealed Copper Scale (IACS), uncertainty, van der pauw, voltmeter linearity.

\section{INTRODUCTION}

$\mathbf{E}$ DDY current conductivity measurements are used as a routine inspection method for vast quantities of aluminum alloys used in aircraft manufacture. Since these conductivity measurements form a very important quality assurance that is directly reflected in the safety of an aircraft, traceability and low uncertainty is of great importance.

The majority of conductivity reference standards produced by manufacturers of eddy current conductivity meters are traceable through conductivity standards measured using a direct current method. This would be a satisfactory situation if the materials had uniform properties throughout their thickness. However, in many cases, the reference standards used in the aerospace industry have a different conductivity in the surface layers compared to that of the bulk of the material, due to the manufacturing process. Since many commercial eddy current conductivity meters operate at a frequency of $60 \mathrm{kHz}$ and above, the penetration depth of the eddy currents is only a few millimeters at most.

In order to solve this unsatisfactory situation, a project is started by three national metrology institutes (NPL, PTB, and $\mathrm{NMi}$ ), two manufacturers of conductivity meters and a representative of the aerospace industry. The project encompasses a thorough investigation of present conductivity measuring methods and a new proposed method, both with direct and alternating current, together with the possible production of a second generation of a wide range of reference materials. One of the project

Manuscript received June 17, 2002; revised October 18, 2002. This work was supported by EU Growth programme under Grant G6RD-CT-2000-00210 ("CONDUCTIVITY" project).

G. Rietveld and C. V. Koijmans are with the Department of Electricity and Magnetism, NMi Van Swinden Laboratorium, Delft, The Netherlands (e-mail: GRietveld@NMi.nl).

L. C. A. Henderson, M. J. Hall, and S. Harmon are with the Centre for Electromagnetic and Time Metrology, NPL, Teddington, U.K.

P. Warnecke and B. Schumacher are with the Abteilung Elektrizität, PTB, Braunschweig, Germany.

Digital Object Identifier 10.1109/TIM.2003.809917

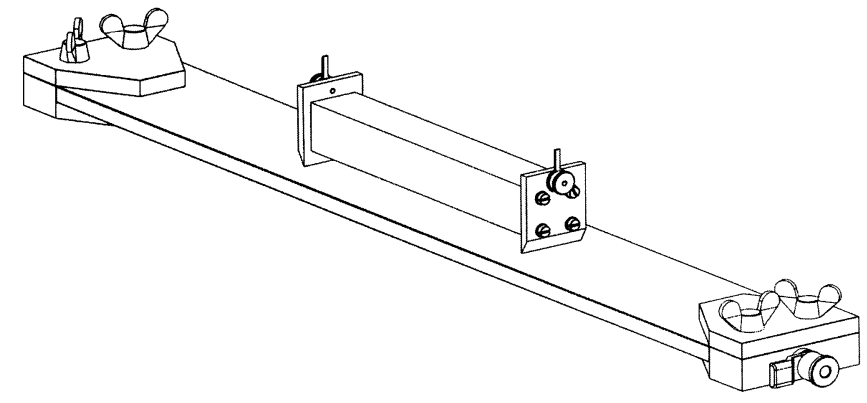

Fig. 1. Measurement configuration of a conventional bar-shaped conductivity sample, with clamps at the end of the bar for feeding the current, and a voltage-sensing element with two knife-edges.

aims is to investigate whether the use of inhomogeneous reference standards in industry is the origin of existing discrepancies between dc and ac techniques.

In this paper, we describe the first results obtained in the project, concerning a new methodology for dc conductivity measurements based on the Van der Pauw technique, which may be useful for ac measurements as well.

\section{MEASUREMENT PRINCIPLE}

The traditional geometry for dc conductivity measurements is shown in Fig. 1. The reference material has a bar shape and the current is applied at the ends using large clamps. The voltage is sensed using two knife-edges that are separated from the main body of the sensing element by Kapton ${ }^{\mathrm{TM}}$ foil. The disadvantage of measurements on bar-shaped references is that, apart from the resistance measurement, a total of three dimensional measurements are needed to obtain the conductivity, namely determination of the distance between the knife-edges and the cross section of the bar. Furthermore, the relatively large size of the bar $(600 \mathrm{~mm} \times 80 \mathrm{~mm} \times 10 \mathrm{~mm})$ makes it more difficult to achieve sufficient homogeneity in the material of the bar. Any inhomogeneity will result in a dependence of the measurement on the position of the voltage knife-edges, and it will furthermore seriously affect eddy current measurements.

Following the initial work of $\mathrm{He}$ et al. [1], a new measurement methodology is investigated using the Van der Pauw technique [2]. See reference [3] for an introduction in Hall effect measurements and resistivity measurements with this technique. In the Van der Pauw geometry, square reference specimens are used which are contacted at the four corners, and the resistance is subsequently measured as indicated in Fig. 2. Two consecutive measurements are performed where the current is applied to two adjacent sides of the sample and the voltage being measured at 


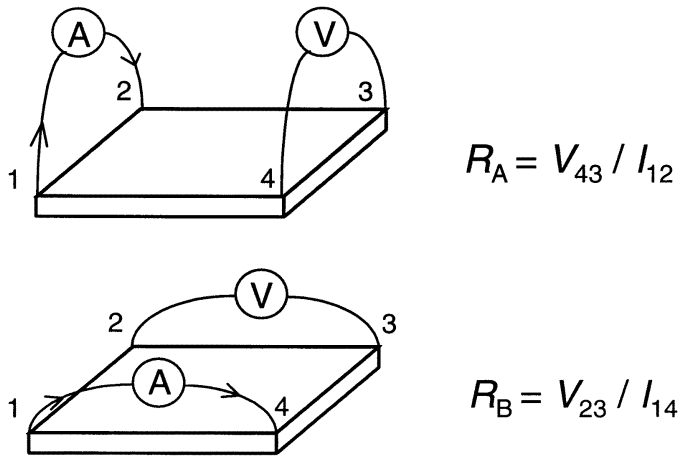

Fig. 2. Measurement of a square conductivity sample in the Van der Pauw geometry. The two resistance measurements are combined with the thickness of the sample to give the conductivity according to (2) [3].

the opposite side of the current contacts. In an argument similar to that of Lampard with respect to the calculable capacitor [4], Van der Pauw proved that from the measured resistance values $R_{A}$ and $R_{B}$ the sheet resistance $R_{S}$ can be derived according to the relation [2]

$$
e^{-\pi R_{A} / R_{S}}+e^{-\pi R_{B} / R_{S}}=1 .
$$

This can be written as

$$
\frac{1}{\sigma}=\frac{\pi d}{\ln (2)} \frac{R_{A}+R_{B}}{2} f(r)
$$

where $d$ is the thickness of the sample and $\sigma=1 / R_{S} \cdot d$ is the required conductivity in MS/m. The function $f(r)$ only depends on the ratio $r=R_{A} / R_{B}$. If $R_{A}$ and $R_{B}$ are equal within $1 \%, f(r)$ is equal to 1 within $0.001 \%$ and can be neglected. Reversing the current and voltage contacts in Fig. 2, we can similarly define $R_{C}$ and $R_{D}$, where, for symmetry reasons, $R_{A}$ should be equal to $R_{C}$ and $R_{B}$ equal to $R_{D}$. The main advantage of the Van der Pauw configuration is that only a single dimensional measurement is needed and the sample is relatively small $(80 \mathrm{~mm} \times 80 \mathrm{~mm} \times 10 \mathrm{~mm})$ so that it is easier to obtain sufficient homogeneity. Also, it gives the possibility of making the measurement on the reference with no transfer stage (at $60 \mathrm{kHz}$ using a commercial eddy current instrument) required.

\section{Measurement SetuP}

The metallic samples that are used as reference standards in conductivity measurements have conductivities of $4 \%$ International Annealed Copper Scale (IACS) to 100\% IACS (with 100\% IACS corresponding to $58.00 \mathrm{MS} / \mathrm{m}$ at $20^{\circ} \mathrm{C}$ ). Given the required 10-mm thickness of the samples, this leads to very low resistances in the Van der Pauw measurements of 0.4 to $10 \mu \Omega$. The main elements of the electrical measurement setup to accurately determine these low resistances are a 1-100 ampere dc current source, reference resistors with several values $(1 \mathrm{~m} \Omega, 100 \mu \Omega$, and $15 \mu \Omega)$, and a nanovolt meter. In the approach followed by NPL, PTB, and He et al. [1], the current source is first calibrated using a reference resistor. Subsequently, the Van der Pauw measurement is performed using the nanovolt meter to measure the voltage across the conductivity sample. This method has the disadvantage that an absolute calibration is needed of the nanovolt meter (typically the 1 $\mathrm{mV}$ range). In another approach followed by $\mathrm{NMi}$, the unknown

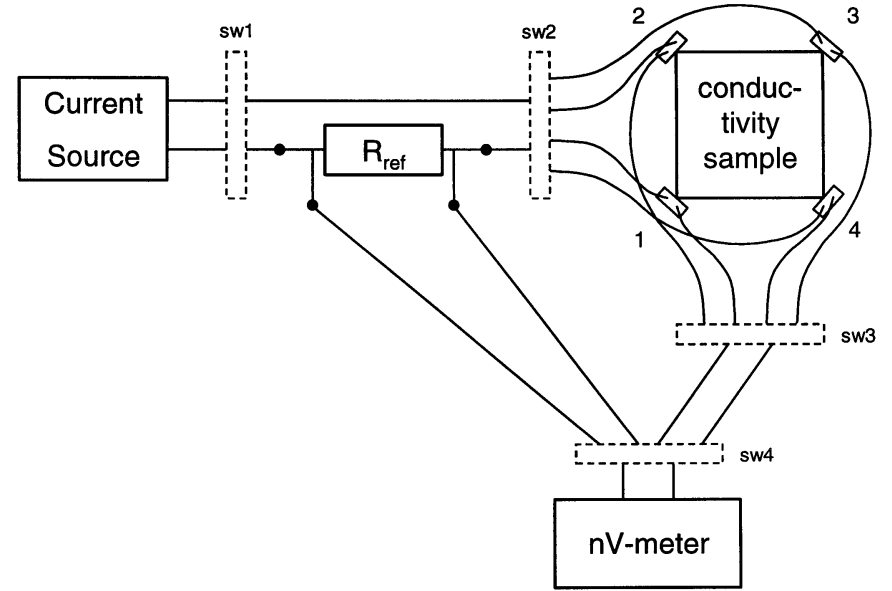

(a)

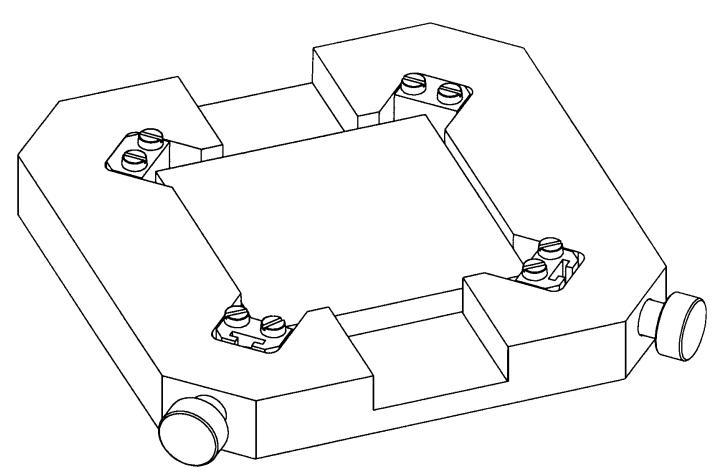

(b)

Fig. 3. (a) Schematic diagram of the setup for conductivity measurements in the Van der Pauw geometry at NMi. The switches sw1-sw4 are for reversing the current polarity and for selecting the current and voltage contacts. The whole setup is computer controlled. (b) Clamp for contacting the corners of the conductivity sample, using four brass blocks and two adjustment screws. Each block has a voltage and current contact.

conductivity sample and a reference resistor are placed in series in the current circuit [see Fig. 3(a)]. The nanovolt meter measures the ratio of the voltages developed across reference resistor and conductivity sample, which is equal to the inverse conductance ratio of reference and sample. Since a ratio measurement is performed, only the linearity of the $1-\mathrm{mV}$ range of the nanovolt meter is important, which is more stable and accurate than the absolute gain.

All instruments in the setup are computer controlled, and the measurements are further automated with an automated switch box, for switching the polarity of the current and for selection of the current and voltage contacts in the Van der Pauw measurements [Fig. 3(a)]. Switching the current polarity is crucial for compensation of thermal voltages.

\section{RESULTS}

An important prerequisite for the validity of formula 2 is that the contact area at the corners of the sample should be small compared to the dimension of the sample. Versnel [5] showed that the error introduced is equal to $2.05 \cdot \lambda^{4}$, with $\lambda$ the ratio between the sum of lengths of the contacts and the length of the boundary of the sample. For our conductivity samples, which are $80 \mathrm{~mm} \times 80 \mathrm{~mm} \times 10 \mathrm{~mm}$, a contact area of $2 \mathrm{~mm}$ thus only introduces an error smaller than $1 \mathrm{ppm}$. Practically, this 
TABLE I

Uncertainty Evaluation of the VAn DER Pauw CONDUCtivity MEASUREMENT AT NMi

The model used for the conductivity $\sigma$ is: $\frac{1}{\sigma}=\rho=\frac{\pi}{\ln (2)} d R_{r e f} r f(r)\left(1+\delta r_{t h}\right)\left(1+\delta r_{l i n}\right)\left(1+\delta r_{T}\right)\left(1+\delta_{\text {cont }}\right)$

\begin{tabular}{|c|c|c|c|c|c|}
\hline Parameter & Value & Uncertainty & $\begin{array}{l}\text { Sensitivity } \\
\text { coefficient }\end{array}$ & \multirow{2}{*}{$\begin{array}{c}\begin{array}{l}\text { Uncertainty } \\
\text { distribution }\end{array} \\
\text { normal }\end{array}$} & \multirow{2}{*}{$\begin{array}{c}\begin{array}{c}\text { Uncer- } \\
\text { tainty } \\
\text { [n } \Omega \mathrm{m}]\end{array} \\
2.78 \cdot 10^{-3}\end{array}$} \\
\hline Thickness $d$ of the sample & $10059.4 \mu \mathrm{m}$ & $1.00 \quad \mu \mathrm{m}$ & $2.78 \cdot 10^{-6} \quad \Omega$ & & \\
\hline Contact size $\delta_{\text {cont }}$ & 0 & $4.62 \cdot 10^{-7}$ & $2.79 \cdot 10^{-2} \quad \mu \Omega \mathrm{m}$ & rectangular & $1.29 \cdot 10^{-5}$ \\
\hline Reference resistor $R_{\text {ref }}$ & $14.9665 \mu \Omega$ & $4.49 \cdot 10^{-4} \mu \Omega$ & $1.87 \cdot 10^{+3} \mu \mathrm{m}$ & normal & $8.38 \cdot 10^{-4}$ \\
\hline Measured ratio $r$ & $4.09317 \cdot 10^{-2}$ & $3.27 \cdot 10^{-6}$ & $6.82 \cdot 10^{-1} \quad \mu \Omega \mathrm{m}$ & normal & $2.23 \cdot 10^{-3}$ \\
\hline Correction factor $\mathrm{f}(\mathrm{r})$ & 1 & $5.77 \cdot 10^{-6}$ & $2.79 \cdot 10^{-2} \quad \mu \Omega \mathrm{m}$ & rectangular & $1.61 \cdot 10^{-4}$ \\
\hline Residual thermal voltages $\delta r_{t h}$ & 0 & $1.15 \cdot 10^{-4}$ & $2.79 \cdot 10^{-2} \quad \mu \Omega \mathrm{m}$ & rectangular & $3.23 \cdot 10^{-3}$ \\
\hline Non-linearity $\mathrm{nV}$-meter $\delta r_{\text {lin }}$ & 0 & $5.77 \cdot 10^{-5}$ & $2.79 \cdot 10^{-2} \quad \mu \Omega \mathrm{m}$ & rectangular & $1.61 \cdot 10^{-3}$ \\
\hline Temperature effect sample $\delta r_{T}$ & $-1.85 \cdot 10^{-4}$ & $8.00 \cdot 10^{-5}$ & $2.79 \cdot 10^{-2} \quad \mu \Omega \mathrm{m}$ & normal & $2.23 \cdot 10^{-3}$ \\
\hline \multirow[t]{2}{*}{ Resistivity $\rho$} & 27.926 & \multicolumn{3}{|c|}{ Total uncertainty: } & $5.60 \cdot 10^{-3}$ \\
\hline & & & \multicolumn{2}{|c|}{ Total expanded uncertainty $(\mathrm{k}=2)$ : } & $1.12 \cdot 10^{-2}$ \\
\hline
\end{tabular}

Final result: conductivity $\sigma=(35.809 \pm 0.014) \mathrm{MS} / \mathrm{m}$

is implemented by pressing brass blocks against the four edges of the corners of the sample as indicated in Fig. 3(b). The adequacy of this method was tested in practice by comparing the results with those obtained on a star shaped sample made from the same batch of material. In this geometry, also used by He et al. [1], the necks of the star produce the required, well-defined current distribution which relaxes the contact geometry requirement. The difference in the results was only $0.025 \%$, well within the measurement repeatability and sample homogeneity of the batch [6]. Note that square samples have the advantage over star shaped samples that they are more compact in size (easier to handle) and much more easily produced. Also, square samples are used in industry as reference for conductivity meters. With the Van der Pauw method, these now can be measured directly by national metrology institutes, i.e., without intermediate transfer measurements.

With the holder of Fig. 3(b), contact resistances as low as $50-100 \mu \Omega$ can be readily obtained. Such low values are important to prevent heating of the contacts by the measurement current. An extensive study was performed to determine the best measurement current: large currents give larger voltage signals but also more heating. In an oil bath with flowing oil operating at $20^{\circ} \mathrm{C}$, the optimal current appeared to be $10 \mathrm{~A}$, where the heating was limited to $30 \mathrm{mK}$. Still, waiting times of a few minutes after selection of new current contacts are needed for thermal voltages to stabilize.

A typical result of a series of Van der Pauw measurements is given in Fig. 4, where all four sides of the sample were measured. It can be seen that $R_{A}=R_{C}$ and $R_{B}=R_{D}$ within $0.002 \%$, far within the noise of the measurement. Furthermore, $R_{A}$ differs only $0.17 \%$ from $R_{B}$ so that the $f(r)$ factor in formula 2 indeed can be neglected. The difference in $R_{A}$ and $R_{B}$ is mainly caused by the nonexact squareness of the sample, but there might be a small effect from nonhomogeneity as well (e.g., different conductivities for directions along and across the rolling direction of the plate from which the sample was machined).

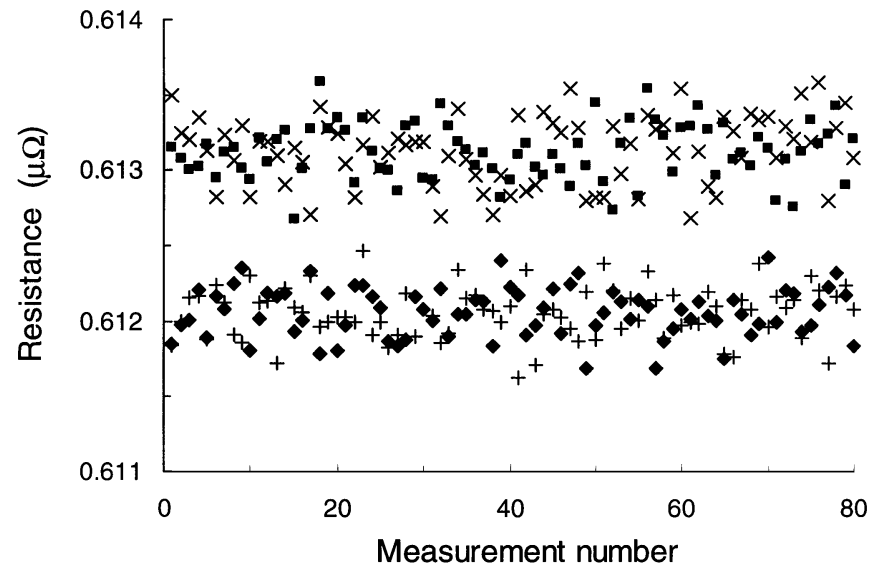

Fig. 4. Typical result of a series of Van der Pauw measurements on a square conductivity sample (results for $R_{A}, R_{B}, R_{C}$, and $R_{D}$ are given by diamonds, squares, plus, and cross symbols, respectively).

The total uncertainty of the measurement is $0.04 \%$ ( $k=$ 2 ), as outlined in Table I. For conductivity values lower than $36 \mathrm{MS} / \mathrm{m}$, the measurement uncertainty is the same, whereas for higher conductivity values, it slightly increases to $0.05 \%$ at $100 \%$ IACS.

An important contribution to the measurement uncertainty is the linearity of the $1-\mathrm{mV}$ range of the nanovolt meter. This was measured in two different ways. First, the $1-\mathrm{mV}$ range was calibrated with the Josephson array voltage standard [Fig. 5(a)]. The deviation from linearity appears to be less than $10 \mathrm{nV}$, that is 10 ppm of the full scale. Second, a ratio measurement was performed of a $1-\mathrm{m} \Omega$ and $100-\mu \Omega$ resistance standard with known values, for different currents and thus for different input voltages at the nanovolt meter. Fig. 5(b) indicates that the deviation from linearity is well within $100 \mathrm{ppm}$ for all relevant input voltages.

As a test of the uncertainty in the Van der Pauw conductivity measurements, three square test samples made from the same sheet of material were measured by the three metrology 


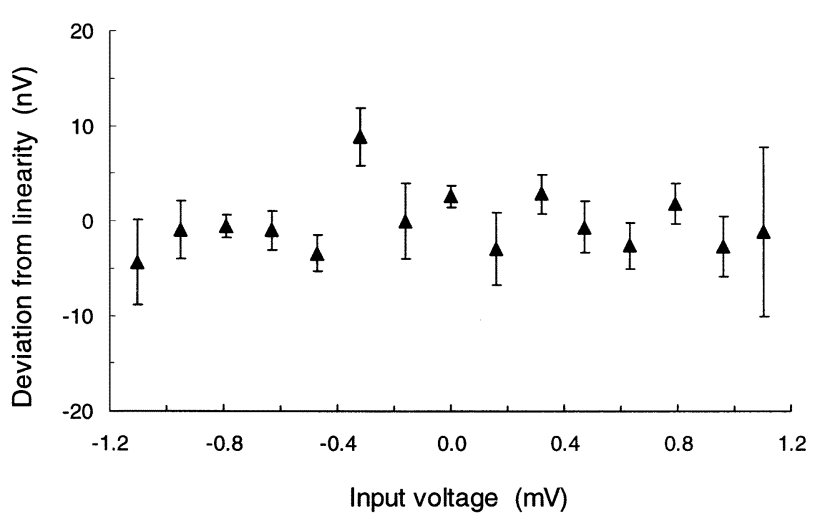

(a)

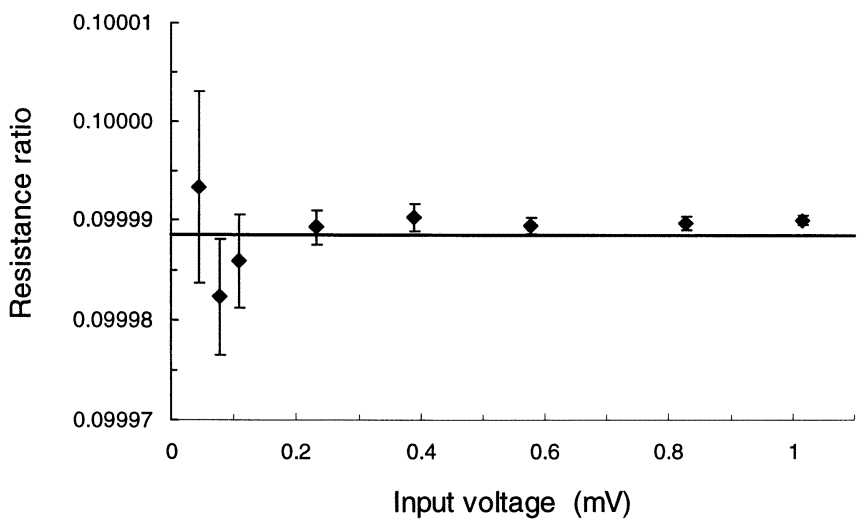

(b)

Fig. 5. Measurement of the linearity of the $1-\mathrm{mV}$ range of the nanovolt meter. (a) Deviation from linearity as a function of input voltage, generated with a Josephson array voltage standard. (b) Measured resistance ratio of a 1-m $\Omega$ and $100-\mu \Omega$ reference resistor as a function of the voltage across the 1-m $\Omega$ resistor. The solid line is based on the known values of the two resistors. Uncertainty bars indicate one standard deviation.

TABLE II

OVERVIEW OF CONDUCTIVITY VALUES OBTAINED BY THREE NATIONAL Metrology Institutes Using a Van der Pauw Method on SQuare Blocks as WELl, AS THE RESUltS OF A MEASUREMENT ON A CONVENTIONAL Bar. All Blocks and Bar are Made From the Same SheEt of Material

\begin{tabular}{c|c|c|c|c}
\hline Institute & Block & $\begin{array}{c}\text { DC } \\
\text { conductivity } \\
\text { (MS/m) }\end{array}$ & $\begin{array}{c}\text { Estimated } \\
\text { Uncertainty } \\
(95 \%)\end{array}$ & $\begin{array}{c}\text { Difference } \\
\text { from average } \\
(\%)\end{array}$ \\
\hline $\mathrm{NPL}$ & 21 & 35.795 & 0.15 & -0.032 \\
\hline $\mathrm{PTB}$ & 23 & 35.815 & 0.06 & +0.024 \\
\hline $\mathrm{NMi}$ & 24 & 35.809 & 0.04 & +0.008 \\
\hline
\end{tabular}

\begin{tabular}{c|c|c|c|c} 
& $\begin{array}{c}\text { Difference } \\
\text { from blocks } \\
(\%)\end{array}$ \\
\hline $\mathrm{NMi}$ & Bar & 35.833 & 0.07 & +0.07 \\
\hline
\end{tabular}

institutes participating in the conductivity project. The resulting values are given in Table II and give an excellent agreement of better than $0.035 \%$. As a second test, a bar from the same material was measured. Also this value agrees well with that of the blocks, namely within $(0.07 \pm 0.07) \%$.

\section{CONCLUSION}

We have successfully implemented and tested a new methodology for conductivity measurements, where square metallic samples are measured in a Van der Pauw geometry. Good quality contacts, with contact resistances less than $100 \mu \Omega$, are made to the samples by pressing blocks against the corners. A comparison of measurement values obtained in this configuration by NPL, PTB, and NMi lead to an agreement of better than $0.035 \%$. A major advantage of the new methodology over conventional conductivity measurements is that smaller samples are required, which can be more easily made with sufficient homogeneity. Also, only a single dimensional measurement is needed instead of the three dimensional measurements in the conventional method. Finally, the reference samples used in industry can now be measured directly at dc, without the need of an intermediate transfer measurement using a commercial eddy current conductivity meter. This reduces the number of possible errors due to $\mathrm{ac} / \mathrm{dc}$ differences. These differences are even stronger reduced by the fact that Van der Pauw measurements are more effectively measuring the in-plane conductivity as sensed by industrial eddy current meters since it involves two resistance measurements in two perpendicular directions, compared to the single measuring direction in the conventional bar measurement. This is especially important when the sample has an in-plane anisotropy, e.g., due to rolling of the plate from which the sample was made.

However, this does not resolve a possible variation in conductivity across the thickness of the sample, which will be subject of further study. Future work will also cover extension of the Van der Pauw method to samples with conductivities covering the range from $2 \%$ IACS to 59\% IACS, and research concentrating on an extension of the Van der Pauw method to ac measurements.

\section{ACKNOWLEDGMENT}

The authors would like to thank the NMi technical support group for help during design and realization of the measurement system at NMi, and J. Bloom and P. C. de Groot for their assistance in the measurements.

\section{REFERENCES}

[1] C. H. He et al., "Cross-conductivity standard for nonferrous materials," IEEE Trans. Instrum. Meas., vol. 44, pp. 181-183, Apr. 1995.

[2] Van der Pauw, "A method of measuring specific resistivity and hall effect of discs of arbitrary shape," Philips Res. Rep., vol. 13, pp. 1-9, 1958

[3] [Online]. Available: http://www.eeel.nist.gov/812/hall.html

[4] D. G. Lampard, Proc. Inst. Elect. Eng. C, vol. 104, no. 6, pp. 271-276, 1957.

[5] W. Versnel, "Analysis of symmetrical Van der Pauw structures with finite contacts," Solid State Electron., vol. 21, pp. 1261-1268, 1978.

[6] L. C. A. Henderson et al., "Toward the resolution of discrepancies in the measurement of low frequency electrical conductivity used for nondestructive testing," in NCSL Int. Workshop Symp., 2002.

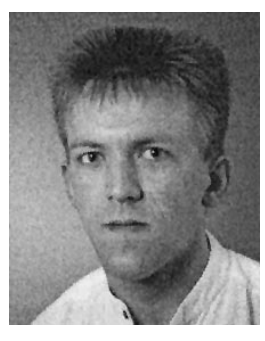

Gert Rietveld was born in The Netherlands in 1965. He received the M.Sc. and Ph.D. degrees in physics from the Delft University of Technology, Delft, The Netherlands, in 1988 and 1993, respectively.

In 1993, he joined the NMi Van Swinden Laboratorium, Delft, where he works in the DC/LF group of the Electrical Metrology Department. He is involved in the development of electrical quantum standards, especially the quantum Hall resistance standard and the current standard based on single electron "self-calibrating" instruments. 
Ch. V. Koijmans, photograph and biography not available at the time of publication.

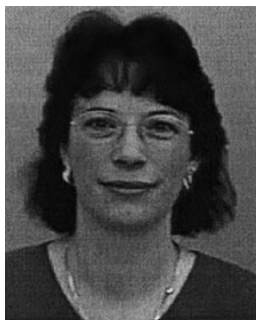

Lesley C. A. Henderson was born in Edinburgh, U.K., in 1959. She received the honors degree in physics from the University of Edinburgh in 1981.

In 1981, she joined the Division of Electrical Science, now the Centre for Electromagnetic and Time Metrology, of the National Physical Laboratory, Teddington, U.K., to work on dc measurement techniques. She developed a system for measuring high value resistors. She then worked on measurement systems using arrays of Josephson junctions, developing primary standards of voltage at $1 \mathrm{~V}$ and $10 \mathrm{~V}$ and was responsible for providing measurement services to industry in $\mathrm{dc}$ voltage before leading a section working on magnetic and transformer measurements, including work on dc and ac conductivity. She is presently Deputy Programme Manager for a program of work on Quantum Metrology.

Ms. Henderson represents the U.K. as EUROMET Contact Person for electricity and is a member of the Technical Advisory Panel of the IEE's Measurement, Sensors, Instrumentation, and NDT Professional Network.

M. J. Hall, photograph and biography not available at the time of publication.

S. Harmon, photograph and biography not available at the time of publication.

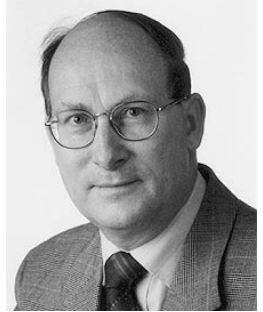

Peter Warnecke was born in Braunschweig, Germany, in 1943. He received the M.S. degree in physics and, in the framework of his work on low-temperature electrical properties of resistance alloys, the Ph.D. degree from the Technische Universität Braunschweig, in 1970 and 1986, respecitvely.

Since 1971, he has been with the PhysikalischTechnische Bundesanstalt (PTB), Braunschweig. Since 1990, he has been the Head of the Laboratory of the Unit of Resistance and, since 1997, he has been the Head of the Section for Direct Current Quantities. He is responsible for the realization and dissemination of the units of voltage and resistance and for calibrations in the field of industrial metrology.

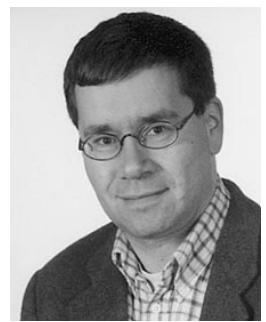

Bernd Schumacher was born in Aachen, Germany, on July 9, 1960. He received the M.S. and Ph.D. degrees from the University of Technology, Aachen, Germany, in 1986 and 1991, respectively.

In 1991, he joined the Physikalisch-Technische Bundesanstalt (PTB), Braunschweig, Germany, where he is currently working on the quantum Hall effect, the CCC, and the calibration of resistance standards. 